\title{
Características clínico epidemiológicas de pacientes con enfermedad crónica en fase terminal admitidos a un departamento de emergencia general

\author{
Clinical and epidemiological characteristics of patients with end-stage chronic disease \\ who were admitted in an emergency department.
}

\author{
José Amado-Tineo ${ }^{1,2, a}$, Rolando Vásquez-Alva ${ }^{1,2, b}$, Roberto Huari-Pastrana ${ }^{1, c}$, Christian Villavicencio-Chávez ${ }^{3,4, \mathrm{~d},}$, Liliana Rimache-Inca ${ }^{1, e}$, Rosa \\ Lizonde-Alejandro, ${ }^{1, f}$ Teodoro Oscanoa-Espinoza, ${ }^{5,6,9}$
}

${ }^{1}$ Departamento de Emergencia, Hospital Nacional Edgardo Rebagliati Martins, Seguro Social de Salud EsSalud. Lima, Perú.

${ }^{2}$ Facultad de Medicina, Universidad Nacional Mayor de San Marcos. Lima, Perú.

${ }^{3}$ Fundación Caredoctors. Barcelona, España.

${ }^{4}$ Facultad de Medicina, Universitat Internacional de Catalunya. Barcelona, España.

${ }^{5}$ Centro de Investigación, Universidad de San Martín de Porres. Lima,Perú.

${ }^{6}$ Departamento de Emergencia, Hospital Nacional Guillermo Almenara Irigoyen, Seguro Social de Salud EsSalud. Lima, Perú.

${ }^{a}$ Médico Internista, Doctor en Medicina. ORCID: https://orcid.org/0000-0002-3286-4650

${ }^{\mathrm{b}}$ Médico Internista, Doctor en Medicina. ORCID: https://orcid.org/0000-0001-6009-7113

${ }^{\circ}$ Médico Emergenciólogo. ORCID: https://orcid.org/0000-0002-7648-6428

${ }^{d}$ Médico Geriatra, Doctor en Medicina. ORCID: https://orcid.org/0000-0002-6433-7265

${ }^{\text {e}}$ Enfermera.

${ }^{\dagger}$ Psicóloga. ORCID: https://orcid.org/0000-0002-5959-6529

${ }_{9}^{9}$ Médico Internista, Doctor en Medicina. ORCID: https://orcid.org/0000-0001-9379-4767

An Fac med. 2020;81(1):21-5 / DOI: https://doi.org/10.15381/anales.v81i1.16848

\begin{abstract}
Correspondencia:
Jose Amado Tineo

jamadot@unmsm.edu.pe
\end{abstract}

Recibido: 10 de octubre 2019 Aceptado: 23 de diciembre 2019

Publicación en línea: 31 de marzo 2020

Conflictos de interés: Los autores declaran no tener conflictos de interés

Fuente de financiamiento: Instituto de Tecnologías en Salud e Investigación, IETSI, Seguro Social de Salud EsSalud, a través del Premio Kaelín 2016.

Contribuciones de autoría: Todos los autores participaron por igual en las etapas de formulación del proyecto, recolección de datos, análisis de datos, redacción y edición del manuscrito, aprobación final del artículo.

El resumen de este artículo fue publicado en el suplemento del congreso de cuidados paliativos realizado en Montreal, Canadá, en octubre de 2018

Citar como: Amado-Tineo J, VásquezAlva R, Huari-Pastrana R, VillavicencioChávez C, Rimache-Inca L, LizondeAlejandro R, et. al. Características clínico epidemiológicas de pacientes con enfermedad crónica en fase terminal admitidos a un departamento de emergencia general. An Fac med. 2020;81 (1):21-5. DOI: https://doi. org/10.15381/anales.v81i1.16848

\section{Resumen}

Introducción. Los pacientes con enfermedad crónica terminal (oncológica y no oncológica) acuden con frecuencia a servicios de urgencias. Objetivos. Identificar enfermedad terminal en pacientes admitidos a emergencia, motivo de ingreso, tratamiento y destino, comparando oncológicos y no oncológicos. Métodos. Estudio observacional en mayores de 18 años admitidos al departamento de emergencia de un hospital terciario. Intrumento: Supportive and Palliative Care Indicators Tool. Análisis estadístico: pruebas de Chi-cuadrado y U Mann-Whitney, considerando significancia $p<0,05$. Resultados. De 4925 admisiones, 271 (5,5\%) cumplieron criterios de enfermedad terminal. Se analizaron 233 pacientes, mediana de edad 77 años [28-99], sexo femenino 59\%. Diagnóstico de cáncer $42 \%$, demencia $23 \%$, secuela neurológica $15 \%$, cirrosis hepática $12 \%$ y otros $8 \%$. Los motivos de ingreso fueron infección $48 \%$, dolor no controlado $9 \%$ y sangrado $7 \%$. Se usó antibióticos en $48 \%$ de pacientes, opioides $12 \%$ (morfina y tramadol) y transfusiones en el 9\%. El 8,6\% recibió soporte vital avanzado (ventilación mecánica 5,6\%, inotrópicos $4,7 \%$ y hemodiálisis $0,8 \%$ ). La mediana de estancia en urgencias fue 6 días [1-62]; falleció 27\%, salió de alta $32 \%$ y $42 \%$ fue derivado a otro servicio hospitalario. La estancia en emergencia y el destino fueron similares en oncológicos y no oncológicos ( $p>0,05)$, presentándose mayor edad y tiempo de enfermedad en pacientes no oncológicos $(p<0,01)$. Conclusiones: Una de cada 20 admisiones al departamento de emergencia evaluado corresponden a enfermedad crónica en fase terminal (más frecuentes no oncológicas), siendo los motivos de ingreso infecciones, dolor y hemorragia.

Palabras clave: Servicios Médicos de Urgencia; Cuidado Terminal; Cuidados Paliativos; Enfermedad Crónica (fuente: DeCS BIREME).

\section{Abstract}

Introduction. Patients with advanced chronic disease (cancer and non-cancer) often go to emergency services. Objectives. To determine terminal chronic disease in those admitted to emergency, reason for admission, treatment and destination, comparing cancer with non-cancer. Methods. Observational study in people over 18 year old admitted to the emergency department of a tertiary hospital. Instrument: Supportive and Palliative Care Indicators Tool. Statistical analysis: Chi-square and U Mann-Whitney tests, considering $p<0.05$. Results. Of 4925 admissions, $271(5,5 \%)$ met criteria. 233 patients were analyzed, median age 77 years [28-99], female sex $59 \%$. Cancer diagnosis $42 \%$, dementia $23 \%$, neurological sequelae $15 \%$, liver cirrhosis $12 \%$ and another $8 \%$. Reason for admission was $48 \%$ infection, $9 \%$ uncontrolled pain and $7 \%$ bleeding. Antibiotics were used in $48 \%$ of patients, opioids $12 \%$ (morphine and tramadol) and transfusions $9 \% ; 8.6 \%$ of these patients received advanced life support (mechanical ventilation $5,6 \%$, inotropic $4,7 \%$ and hemodialysis $0,8 \%$ ). The median stay in the emergency room was 6 days [1-62]; $27 \%$ died, $32 \%$ were discharged and $42 \%$ were referred to another hospital department. The stay and reason for admission were similar in cancer and non-cancer $(p>0,05)$, presenting older age and disease time in non-cancer patients $(p<0,01)$. Conclusions. One of every 20 admissions to the emergency department evaluated corresponds to a chronic disease in the terminal phase (more frequent non-cancer), being the reasons for admission infections, pain and bleeding.

Keywords: Emergency Medical Services; Terminal Care; Palliative Care; Chronic Disease (source: MeSH NLM). 


\section{INTRODUCCIÓN}

El servicio de urgencias o emergencias de un hospital se encuentra diseñado y equipado para brindar atención inmediata a pacientes con problemas de salud que pongan en riesgo su vida o produzcan secuelas invalidantes; siendo el fin principal salvar la vida ${ }^{(1)}$. En las últimas décadas, la sobredemanda de atención debido a incremento poblacional (por avance tecnológico y transición epidemiológica), ha producido saturación de estos servicios, principalmente en los grandes hospitales de referencia, predominando pacientes de edad avanzada con enfermedades crónicas y complejas ${ }^{(2,3)}$.

Se define como enfermedad crónica en fase terminal a la presencia de daños orgánicos irreversibles, múltiple sintomatología, pronóstico de vida limitado y gran impacto emocional para el paciente, la familia y el personal de salud; recomendándose los cuidados paliativos como medida más efectiva, así como evitar ciertos procedimientos invasivos al paciente que prolongan su sufrimiento. El término "enfermedad terminal" es cuestionado actualmente (por posible estigmatización del paciente) y en su lugar se prefiere hablar de necesidad de cuidados paliativos, para lo cual se han desarrollado diversas herramientas ${ }^{(4,5,6)}$.

En Canadá el 57,6\% de pacientes con cáncer visitaron el servicio de emergencia en su último mes de vida (7); en Australia el 52\% de los mayores de 18 años fallecidos por cualquier causa también acudieron a emergencia en el mismo periodo ${ }^{(8)}$ y en Estados Unidos de Norteamérica hasta $16 \%$ de los mayores de 65 años que fueron admitidos a un servicio de urgencias por cualquier patología tenía decisión expresa de limitar soporte (orden de no reanimación) ${ }^{(9)}$. Sin embargo, la frecuencia de este evento es subestimada ${ }^{(10,11)}$. También se ha reportado que el empleo de cuidados paliativos en este tipo de pacientes (institucionalizados o con seguimiento domiciliario), disminuye los ingresos hospitalarios, ingresos en unidades de cuidados intensivos y uso frecuente del servicio de urgencias; sin diferencia significativa con el tiempo de supervivencia ${ }^{(12,13,14)}$. Pero también se reporta que estos pacientes fallecen en urgencias sin recibir cuidados paliativos ${ }^{(15,16)}$. No se encontraron investigaciones publicadas sobre el tema en otros países de Latinoamérica.

En hospitales urbanos de Perú existe hacinamiento de los servicios de emergencia ${ }^{(2,17)}$, reportándose en uno de ellos que el $10 \%$ de las admisiones a emergencia presentaba una enfermedad en fase terminal, asociándose a estancia hospitalaria prolongada, readmisión frecuente y elevada tasa de mortalidad ${ }^{(3,18)}$. Por tal motivo, el presente estudio tuvo como objetivo identificar la frecuencia y características de pacientes con enfermedad crónica terminal admitidos al departamento de emergencia de un hospital nacional e identificar motivo de ingreso, tratamiento recibido y destino, comparando patología oncológica y no oncológica.

\section{MÉTODOS}

\section{Diseño del estudio}

Estudio observacional, en pacientes mayores de 18 años de edad admitidos al Servicio de Emergencia del Hospital Nacional Edgardo Rebagliati Martins, del Seguro Social de Salud EsSalud, Lima, Perú, el cual cuenta con unidad de trauma shock, tópicos de atención (medicina, cirugía y traumatología), y 200 camillas de observación.

\section{Población y muestra}

Se revisaron todas las admisiones al servicio de emergencia entre 1 으 de febrero y 30 de abril de 2017, considerando enfermedad crónica en fase terminal a los que cumplian indicadores clínicos asociados al final de la vida del Supportive and Palliative Care Indicators Tool (SPICT-ESTM) 2015, adaptada y validada en español ${ }^{(19)}$. Se excluyeron los casos con datos incompletos, readmisiones, fallecidos o transferidos durante las primeras 24 horas de admisión.

EI SPICT-ESTM fue desarrollado inicialmente, en inglés, por la Universidad de Edimburgo (2010) como una herramienta concisa y sencilla para identificar a los pacientes con enfermedades crónicas que necesitaban cuidados paliativos, incluye 27 ítems de respuesta dicotómica (si/no) agrupados en dos categorías: indicadores generales de deterioro de la salud e indicadores clínicos de enfermedad avanzada. Ha sido validada en idioma español demostrando adecuada consistencia interna y confiabilidad (índice Kappa entre 0,983 y 0,797 para sus diferentes ítems) ${ }^{(19)}$. Se puede acceder a este instrumento en la siguiente dirección electrónica: http:// www.spict.org.uk/the-spict/spict-es/spictes-download/.

\section{Variables de estudio}

Se registró la etiología de la enfermedad crónica, motivo de consulta, tratamiento recibido en el departamento de emergencia y procedimientos invasivos probablemente innecesarios según consenso del Colegio Australiano de Medicina de Emergencia ${ }^{(6)}$.

Se revisaron reportes médicos diarios, historia clínica y se realizaron entrevistas y evaluaciones al paciente o cuidador principal, por médicos especialistas del servicio de urgencias, capacitados en criterios de terminalidad en enfermedad crónica. Posteriormente se determinó el destino final en el sistema estadístico del hospital.

\section{Análisis estadístico}

Los datos fueron registrados en una ficha prediseñada, codificados, digitados en Microsoft Excel $2010{ }^{\circledR}$ y procesados con IBM SPSS Statistics for Windows, Version 24.0 (IBM Corp. Released $2016{ }^{\circledR}$ ). Para comparar los grupos oncológicos y no oncológicos, se aplicó análisis bivariado (en casos con aceptable potencia estadística) cualitativo con prueba de Chi cuadrado y U de Mann-Whitney para muestras independientes de distribución no normal, considerando significativo $\mathrm{p}<0,05$.

\section{Aspectos éticos}

Los pacientes fueron evaluados siguiendo los principios de buenas prácticas clínicas y la declaración de Helsinki. Esta investigación se obtuvo de la fase inicial de un protocolo de investigación sobre impacto de cuidados paliativos en emergencia, aprobado por el Comité Hospita- 
lario de Ética en Investigación del Hospital Nacional Edgardo Rebagliati Martins.

\section{RESULTADOS}

De 4925 admisiones durante el periodo evaluado, 271 (5,5\%) cumplieron criterios planteados. Se excluyeron 38 admisiones (25 por datos incompletos, 10 por readmisión durante el periodo de estudio y tres por fallecimiento). Se analizaron 233 pacientes con una edad media de 77 años (entre 28 y 99 ) y de las que un $59 \%$ perteneció al sexo femenino. El 58\% tenían patología no oncológica y $42 \%$ presentaron algún tipo de cáncer. Los sitios primarios más frecuentes en pacientes oncológicos fueron: mama, pulmón, colon, hígado y páncreas, mientras que las etiologías no oncológicas: secuela de enfermedad cerebrovascular, demencia, fibrosis pulmonar, cirrosis hepática por alcohol y cardiopatía isquémica (Figura 1).
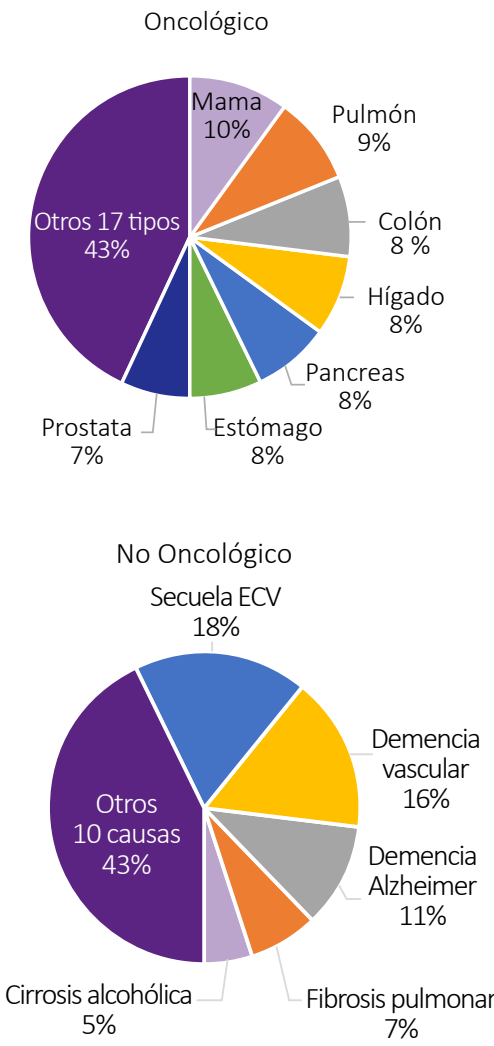

Figura 1. Etiologia de enfermedad crónica en fase terminal de pacientes admitidos al Servicio de Emergencia del Hospital Nacional Edgardo Rebagliati Martins, Febrero - Abril, 2017.
La mayoría procedía de su domicilio (85\%). Fueron admitidos en shock trauma (44\%) y el tópico de medicina (50\%). Se observaron diferencias significativas entre la edad, el tiempo de enfermedad y el tiempo de enfermedad terminal, entre pacientes con patología oncológica y no oncológica (Tabla 1). Los motivos de ingreso fueron procesos infecciosos en un 48\%: 57 casos con patología respiratoria, 29 con infección urinaria, nueve con procesos digestivos y siete con infecciones dérmicas; seguidos por dolor no controlado en un 9\% (14 casos de dolor abdominal, tres de dolor lumbar, dos de dolor torácico y un caso de cefalea) y sangrado 7\% (11 casos de sangrado digestivo alto, dos, de sangrado digestivo bajo, un caso de hematuria, uno de epistaxis, uno de hemoptisis y uno de sangrado tumoral).

El destino de los pacientes con enfermedad en fase terminal al egreso de emergencia fue: alta 31\%, fallecido $27 \%$ y otra sala del hospital $42 \%$ (Figura 2), similar en ambos grupos. La mediana de estancia en salas de emergencia fue seis días (de 1 a 62 días), similar en oncológicos y no oncológicos $(p=0,91)$. Los pacientes que fueron derivados a otras salas hospitalarias después de emergencia, tuvieron 17 días como mediana de estancia (rango intercuartílico 15,5), falleciendo en dichas salas el $45 \%$ de ellos.

El procedimiento invasivo más frecuentemente realizado a pacientes con enfermedad crónica en fase terminal, admitidos en el servicio de urgencias del hospital evaluado, fue la colocación de sonda nasogástrica (Tabla 2). Por otro lado, con respecto al tratamiento brindado, 8,6\% de estos pacientes recibió soporte vital avanzado (ventilación mecánica 5,6\%, inotrópicos $4,7 \%$ y hemodiálisis $0,8 \%$ ). Asimismo, el $48 \%$ de los pacientes recibió tratamiento con antimicrobianos, mientras que el 12\%, recibió opioides (Tabla 3).

Tabla 1. Características de los pacientes con enfermedad crónica en fase terminal admitidos al Servicio de Emergencia del Hospital Nacional Edgardo Rebagliati Martins. Febrero - Abril, 2017.

\begin{tabular}{|c|c|c|c|c|}
\hline Características & $\begin{array}{l}\text { Oncológicos } \\
(n=99)\end{array}$ & $\begin{array}{l}\text { No Oncológicos } \\
(n=134)\end{array}$ & $\begin{array}{c}\text { Total } \\
(n=233)\end{array}$ & Valor $p$ \\
\hline $\begin{array}{l}\text { Edad en años: mediana }\left(\mathrm{RI}^{\&}\right) \\
\text { Rango }\end{array}$ & $\begin{array}{l}68(23) \\
35-90\end{array}$ & $\begin{array}{l}82(16) \\
28-99\end{array}$ & $\begin{array}{l}77(21) \\
28-99\end{array}$ & $<0,01^{*}$ \\
\hline Sexo Femenino & $58(58,6 \%)$ & $79(59,0 \%)$ & $137(58,8 \%)$ & $N A^{* *}$ \\
\hline $\begin{array}{l}\text { En servicios paliativos: } \\
\text { COPHOES\# } \\
\text { PADOMI }\end{array}$ & $\begin{array}{c}14(14,1 \%) \\
12(12,1 \%) \\
2(2,0 \%)\end{array}$ & $\begin{aligned} 28 & (20,9 \%) \\
1 & (0,7 \%) \\
27 & (20,1 \%)\end{aligned}$ & $\begin{array}{c}42(18,0 \%) \\
13(5,6 \%) \\
29(12,4 \%)\end{array}$ & NA \\
\hline $\begin{array}{l}\text { Lugar de procedencia: } \\
\text { - Domicilio } \\
\text { - Casa de reposo } \\
\text { - Hospital o Clínica }\end{array}$ & $\begin{array}{c}88(88,9 \%) \\
0(0,0 \%) \\
8(8,1 \%)\end{array}$ & $\begin{array}{c}108(80,6 \%) \\
20(14,9 \%) \\
6(4,5 \%)\end{array}$ & $\begin{array}{c}196(84,1 \%) \\
20(8,6 \%) \\
15(6,4 \%)\end{array}$ & NA \\
\hline $\begin{array}{l}\text { Área de admisión: } \\
\text { - Shock Trauma } \\
\text { - Medicina } \\
\text { - Cirugía/Traumatología }\end{array}$ & $\begin{array}{c}38(38,4 \%) \\
53(53,5 \%) \\
8(8,1 \%)\end{array}$ & $\begin{array}{c}65(48,5 \%) \\
64(47,8 \%) \\
5(3,7 \%)\end{array}$ & $\begin{array}{c}103(44,2 \%) \\
117(50,2 \%) \\
13(5,6 \%)\end{array}$ & NA \\
\hline $\begin{array}{l}\text { Motivo de ingreso: } \\
\text { - Infección } \\
\text { - Dolor } \\
\text { - Encefalopatía } \\
\text { - Sangrado } \\
\text { - Disnea }\end{array}$ & $\begin{array}{c}31(31,3 \%) \\
17(17,2 \%) \\
2(2,0 \%) \\
10(10,1 \%) \\
7(7,1 \%)\end{array}$ & $\begin{array}{c}80(59,7 \%) \\
3(2,2 \%) \\
17(12,7 \%) \\
7(5,2 \%) \\
8(6,0 \%)\end{array}$ & $\begin{array}{c}111(47,6 \%) \\
20(8,6 \%) \\
19(8,2 \%) \\
17(7,3 \%) \\
15(6,4 \%)\end{array}$ & NA \\
\hline $\begin{array}{l}\text { Tiempo de enfermedad en } \\
\text { meses }(n=134)\end{array}$ & $\begin{array}{c}12(33) \\
1-180\end{array}$ & $\begin{array}{c}32(51) \\
1-456\end{array}$ & $\begin{array}{c}14(42) \\
1-456\end{array}$ & $<0,01^{*}$ \\
\hline $\begin{array}{l}\text { Tiempo Enfermedad avanzada } \\
\text { en meses }(n=137)\end{array}$ & $\begin{array}{l}3(4) \\
1-48\end{array}$ & $\begin{array}{l}8(8) \\
1-48\end{array}$ & $\begin{array}{c}5(10) \\
1-48\end{array}$ & $<0,01^{*}$ \\
\hline
\end{tabular}

\& RI: rango intercuartílico, \# COPHOES: servicio de control post hospitalario especializado, \$ PADOMI: Programa de atención domiciliaria, * Prueba U de Mann-Whitney, ** NA: no aplica 


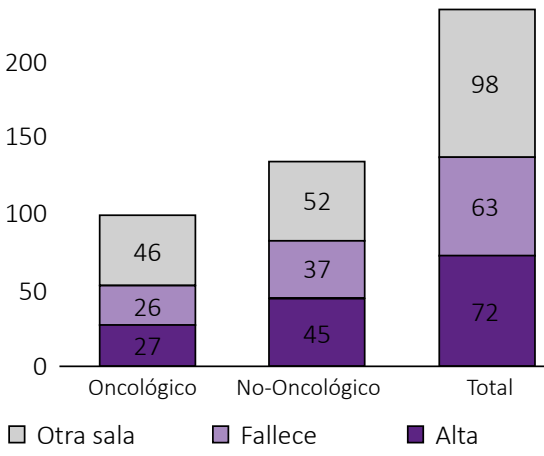

Figura 2. Destino de pacientes con enfermedad en fase terminal admitidos al Servicio de Emergencia del Hospital Nacional Edgardo Rebagliati Martins. Febrero - Abril, 2017.

\section{DISCUSIÓN}

Según nuestros hallazgos, el 5,5\% de las admisiones al servicio de emergencia de un hospital terciario de la seguridad social corresponden a pacientes con enfermedad en fase terminal, siendo más frecuentes la patología no oncológica. Esta cifra es menor a lo reportado previamente en nuestro país, probablemente por el instrumento utilizado o el número de pacientes evaluados; pero es similar a lo reportado en algunos países de Europa, destacando que no existen reportes suficientes a nivel mundial ${ }^{(4,11,18,20)}$.

Las enfermedades no oncológicas, como insuficiencias orgánicas o secuelas neurodegenerativas, fueron más frecuentes; sin embargo, a nivel mundial son menos estudiadas que las patologías oncológicas, a pesar de presentar mayor sobrevida y uso de servicios de salud. En este estudio llama la atención la baja frecuencia de cardiopatías. Probablemente este grupo de pacientes fue catalogado como secuelas de enfermedad cerebrovascular, que son complicaciones de enfermedad cardíaca avanzada ${ }^{(21,22)}$.

Más del $40 \%$ de estos pacientes ingresó al departamento de emergencias por la unidad de shock trauma, área de la emergencia destinada a la reanimación del paciente crítico. Los motivos de ingreso encontrados fueron similares a otro estudio destacando las infecciones ${ }^{(23)}$. Se encontró mayor edad, tiempo de enfermedad, complicación infecciosa, procedencia de casa de reposo (hospicio) y uso previo de servicios paliativos en los pacientes con patología avanzada no oncológica. Mien-

Tabla 2. Procedimientos invasivos realizados en pacientes con enfermedad crónica en fase terminal admitidos al Servicio de Emergencia del Hospital Nacional Edgardo Rebagliati Martins. Febrero Abril, 2017.

\begin{tabular}{lccc} 
Procedimiento & $\begin{array}{c}\text { Oncológicos } \\
(\mathbf{n = 9 9 )}\end{array}$ & $\begin{array}{c}\text { No Oncológicos } \\
\mathbf{( n = 1 3 4 )}\end{array}$ & $\begin{array}{c}\text { Total } \\
\text { (n=233) }\end{array}$ \\
\hline Sonda nasogástrica & $13(13,1 \%)$ & $66(49,3 \%)$ & $79(33,9 \%)$ \\
\hline Intubación orotraqueal & $4(4,0 \%)$ & $15(11,2 \%)$ & $19(8,2 \%)$ \\
\hline Inotrópicos por CVC \& & $11(11,1 \%)$ & $7(5,2 \%)$ & $18(7,7 \%)$ \\
\hline Ventilación mecánica invasiva & $3(3,0 \%)$ & $12(9,0 \%)$ & $15(6,4 \%)$ \\
\hline Hemodiálisis & $1(1,0 \%)$ & $4(3,0 \%)$ & $5(2,1 \%)$ \\
\hline Transfusiones & $15(15,2 \%)$ & $9(6,7 \%)$ & $24(10,3 \%)$ \\
\hline
\end{tabular}

\& CVC: catéter venoso central

tras que en los pacientes oncológicos se reportó mayor frecuencia de dolor y sangrado $(10,15,24,25)$.

La estancia media en urgencias fue similar en ambos grupos, pero mayor a lo recomendado para este servicio, debido probablemente a deficiencias del sistema de salud ${ }^{(2)}$. También se destaca que alrededor del $40 \%$ de estos pacientes con enfermedad en fase terminal, fueron derivados a otros servicios de hospitalización, donde también permanecieron un tiempo prolongado y casi la mitad finalmente falleció; esto expresa la necesidad de los correspondientes cuidados paliativos ${ }^{(25)}$.

Se encontró un porcentaje importante de procedimientos invasivos considerados innecesarios, probablemente debido a desconocimiento o escasa capacitación del personal de salud en cuidados paliativos, exigencia de la familia o vacíos legales en la región ${ }^{(5,6,15,26)}$. Se administró tratamiento antibiótico en casi la mitad de los casos, de mayor complejidad en los pacientes oncológicos. La terapia analgésica fue poco utilizada y la proporción de opioides y morfina también fue baja, incluso en pacientes oncológicos. Esto pue- de asociarse a la baja frecuencia de dolor como motivo de consulta, pero también al cuidado por parte de los médicos para usar estos fármacos ${ }^{(27)}$. Así, se realizaron procedimientos probablemente innecesarios en la quinta parte de estos, con estancia prolongada y alta tasa de fallecimiento hospitalario.

La alta demanda de atención en los servicios de emergencia de hospitales de tercer nivel de atención de salud, refleja problemas en el sistema nacional de salud, con deficiencia en el enfoque de atención en cuidados paliativos, siendo parte de la solución mejorar la selección de pacientes y referirlos a servicios adecuados, evitando procedimientos innecesarios y mejorando su calidad de vida más que prolongar su sufrimiento ${ }^{(28,29)}$. Esto plantea la necesidad de integrar los cuidados paliativos al servicio de emergencia, como se viene haciendo en otros países ${ }^{(4,16,21,30)}$.

La atención de pacientes con enfermedades crónicas avanzadas en los servicios de emergencia es un tema complejo desde el punto de vista ético y en el país no existen bases legales claras. Sin em-

Tabla 3. Tratamiento farmacológico en pacientes con enfermedad crónica en fase terminal admitidos al Servicio de Emergencia del Hospital Nacional Edgardo Rebagliati Martins. Febrero - Abril, 2017.

\begin{tabular}{lccc} 
Tratamiento & $\begin{array}{c}\text { Oncológicos } \\
(\mathbf{n}=\mathbf{9 9})\end{array}$ & $\begin{array}{c}\text { No Oncológicos } \\
\mathbf{( n = 1 3 4 )}\end{array}$ & $\begin{array}{c}\text { Total } \\
(\mathbf{n}=\mathbf{2 3 3})\end{array}$ \\
\hline Antimicrobianos & $31(31,3 \%)$ & $80(59,7 \%)$ & $111(47,6 \%)$ \\
- Ceftriaxona + clindamicina & $10(10,1 \%)$ & $40(29,9 \%)$ & $50(21,5 \%)$ \\
- Imipenem + vancomicina & $16(16,2 \%)$ & $10(7,5 \%)$ & $26(11,2 \%)$ \\
\hline Opioides: & $26(26,3 \%)$ & $2(1,5 \%)$ & $28(12,0 \%)$ \\
- Morfina & $14(14,1 \%)$ & $0(0,0 \%)$ & $14(6,0 \%)$ \\
- Tramadol & $12(12,1 \%)$ & $2(1,5 \%)$ & $14(6,0 \%)$ \\
\hline Metamizol & $5(5,1 \%)$ & $11(8,2 \%)$ & $16(6,9 \%)$ \\
\hline Haloperidol & $1(1,0 \%)$ & $10(7,5 \%)$ & $11(4,7 \%)$ \\
\hline
\end{tabular}


bargo, la adecuada aplicación de cuidados paliativos mejoraría la calidad de vida del paciente y a la vez permitiría que ambientes de áreas críticas se encuentren disponibles para atenciones de pacientes con otras patologías y necesidades.

Entre las limitaciones del presente estudio, se realizó en un solo hospital con hacinamiento de pacientes, perteneciente a la seguridad social (que incluye aproximadamente al $40 \%$ de la población nacional), durante un corto periodo de tiempo, y con una muestra no probabilística. No existe legislación clara en nuestro medio respecto a decisiones al final de la vida y cuidados paliativos, existiendo dudas en la toma de decisiones. Asimismo, la subjetividad de la información brindada.

Concluimos que una de cada 20 admisiones al servicio de emergencia del hospital evaluado presentó enfermedad crónica en fase terminal, con mayor frecuencia no oncológica, siendo los motivos de ingreso infecciones, dolor no controlado y hemorragias.

\section{REFERENCIAS BIBLIOGRÁFICAS}

1. Ministerio de salud. Norma Técnica de los Servicios de Emergencia de Hospitales del Sector Salud. Lima-Perú; 2004.

2. Vásquez Alva R, Amado Tineo J, Ramírez Calderón $F$, Velásquez Velásquez $R$, Huari Pastrana $R$. Sobredemanda de atención médica en el servicio de emergencia de adultos de un hospital terciario, Lima, Perú. An Fac Med. 2016;77(4):379. DOI: 10.15381/anales.v77i4.12654

3. Amado J, Vasquez R, Huari R, Palacios A. Readmision frecuente de pacientes al servicio de emergencia de adultos de un hospital terciario. Lima-Peru 2012. Rev Soc Peru Med Interna. 2015;28(4):153-7. DOI: 10.13140/rg.2.1.2230.5041.

4. George N, Phillips E, Zaurova M, Song C, Lamba S, Grudzen C. Palliative Care Screening and Assessment in the Emergency Department: A Systematic Review. J Pain Symptom Manage. 2016;51(1):108119.e2. DOI: 10.1016/j.jpainsymman.2015.07.017

5. World Health Organization (WHO). Global Atlas of Palliative Care at the End of Life. Geneva, Switzerland; 2014

6. Australasian College for Emergency Medicine. Policy on end of life and palliative care in the emergency department (P455). Emerg Med Australas. 2016;28(5):617-21. DOI: 10.1111/1742-6723.12674

7. Bekelman JE, Halpern SD, Blankart CR, Bynum JP, Cohen J, Fowler R, et al. Comparison of Site of Death, Health Care Utilization, and Hospital Expenditures for Patients Dying With Cancer in
7 Developed Countries. JAMA. United States; 2016;315(3):272-83. DOI: 10.1001/jama.2015.18603

8. Goldsbury DE, O'Connell DL, Girgis A, Wilkinson A, Phillips JL, Davidson PM, et al. Acute hospital-based services used by adults during the last year of life in New South Wales, Australia: a population-based retrospective cohort study. BMC Health Serv Res. England; 2015;15:537. DOI: 10.1186/s12913-0151202-8

9. Richardson DK, Zive DM, Newgard CD. End-of-life decision-making for patients admitted through the emergency department: hospital variability, patient demographics, and changes over time. Acad Emerg Med Off J Soc Acad Emerg Med. United States; 2013;20(4):381-7. DOI: 10.1111/acem.12112

10. da Silva Soares D, Nunes CM, Gomes B. Effectiveness of Emergency Department Based Palliative Care for Adults with Advanced Disease: A Systematic Review. J Palliat Med. 2016;19(6):601-9. DOI: 10.1089/jpm.2015.0369

11. Wong J, Gott M, Frey R, Jull A. What is the incidence of patients with palliative care needs presenting to the Emergency Department? A critica review. Palliat Med. 2014;28(10):1197-205. DOI: $10.1177 / 0269216314543318$

12. Quiñones Pérez A, Álvarez Jiménez P, Garcia Sánchez MJ, Guardia Mancilla P. Influencia de la atención avanzada en cuidados paliativos en la frecuentación de las urgencias hospitalarias. Med Paliativa. 2013;20(2):60-3. DOI: 10.1016/j. medipa.2012.07.003

13. Ali AA, Adam R, Taylor D, Murchie P. Use of a structured palliative care summary in patients with established cancer is associated with reduced hospital admissions by out-of-hours general practitioners in Grampian. BMJ Support Palliat Care. England; 2013;3(4):452-5. DOI: 10.1136/bmjspcare-2012-000371

14. Liu T, Chen J, Wang H, Wu S, Hung Y, ST T. Symptom management and supportive care. Quality of end-of-life care between medical oncologists and other physician specialists for Taiwanese cancer decedents, 2001-2006. Oncologist. National Institute of Cancer Research, National Health Research Institutes, Miaoli County, Taiwan.: AlphaMed Company, Inc., dba AlphaMed Press; 2009;14(12):1232-41. DOI: 10.1634/theoncologist.2009-0095

15. Metola Gómez A, García Romo E, Bellido De Vega S, Carretero Lanchas Y, Azcoitia Álvarez B, Guerrero L. Análisis de la evolución de pacientes en probable situación de últimos dias en un servicio de Urgencias. Med Paliativa. 2013;20(1):10-8. DOI: 10.1016/j.medipa.2012.01.011

16. Liberman T, Kozikowski A, Kwon N, Emmert B, Akerman M, Pekmezaris R. Identifying Advanced IIIness Patients in the Emergency Department and Having Goals-of-Care Discussions to Assist with Early Hospice Referral. J Emerg Med. 2018;54(2):191-7. DOI: 10.1016/j.jemermed.2017.08.009

17. Taype-Huamani W, Chucas-Ascencio L, AmadoTineo J. Hacinamiento del servicio de emergencia de adultos del hospital Rebagliati: Reporte de caso. Rev Med Rebagliati. 2018;1(2):50-5.

18. Amado-Tineo J, Taype-Huamani W, Vasquez-alva R, Campo FS, Oscanoa-espinoza T. Pacientes con enfermedad crónica terminal en el servicio de emergencia de adultos de un hospital terciario. Rev
Fac Med Hum. 2019;19(3):27-34. DOI: 10.25176/ RFMH.v19i3.2166

19. Fachado AA, Martínez NS, Roselló MM, Ria JJV, Oliver EB, García RG, et al. Spanish adaptation and validation of the supportive \&amp; palliative care indicators tool - SPICT-ES ${ }^{T M}$. Rev Saúde Pública. 2018;52:3. DOI: 10.11606/S15188787.2018052000398

20. Le Conte P, Riochet D, Batard E, Volteau C, Giraudeau $B$, Arnaudet I, et al. Death in emergency departments: a multicenter cross-sectional survey with analysis of withholding and withdrawing life support. Intensive Care Med. 2010;36(5):765-72.

21. Glajchen M, Lawson R, Homel P, Desandre P, Todd $\mathrm{KH}$. A rapid two-stage screening protocol for palliative care in the emergency department: a quality improvement initiative. J Pain Symptom Manage. 2011;42(5):657-62. DOI: 10.1016/j.jpainsymman.2011.06.011

22. Rosenwax LK, Mcnamara BA, Murray K, Mccabe RJ, Aoun SM, Currow DC. Hospital and emergency department use in the last year of life: a baseline for future modifications to end-of-life care | Medical Journal of Australia. Med J Aust. 2011;194(11):570-3. DOI: 11.5694/mja11.11173

23. Alsirafy SA, Raheem AA, Al-Zahrani AS, Mohammed AA, Sherisher MA, El-Kashif AT, et al. Emergency Department Visits at the End of Life of Patients With Terminal Cancer: Pattern, Causes, and Avoidability. Am J Hosp Palliat Med. 2016;33(7):658-62. DOI: 10.1177/1049909115581819

24. Van Tricht M, Riochet D, Batard E, Martinage A, Montassier E, Potel G, et al. Palliative care for patients who died in emergency departments: analysis of a multicentre cross-sectional survey. Emerg Med J EMJ. 2012;29(10):795-7. DOI: 10.1136/ emermed-2011-200513

25. Amado JP, Vasquez R, Huari RW, Sucari AS, Oscanoa TJ. Patients with End-stage Oncologic and Nononcologic Disease in Emergency Service of an Urban Tertiary Hospital. Indian J Palliat Care. India; 2018;24(1):25-7. DOI: 10.4103/IJPC.IJPC_108_17

26. Lafond P, Chalayer E, Roussier M, Weber E, Lacoin-Reynaud Q, Tardy B. A Hospice and Palliative Care Bed Dedicated to Patients Admitted to the Emergency Department for End-of-Life Care. Am J Hosp Palliat Med. 2016;33(4):403-6. DOI: 10.1177/1049909114562947

27. Bonilla P, De Lima L, Díaz P, León MX, González M. Uso de Opioides en tratamiento del DOLOR. Manual para Latinoamérica. Primera. Caracas, Venezuela: Association for Hospice and Palliative Care; 2011.

28. Smith AK, McCarthy E, Weber E, Cenzer IS, Boscardin J, Fisher J, et al. Half Of Older Americans Seen In Emergency Department In Last Month Of Life; Most Admitted To Hospital, And Many Die There. Health Aff (Millwood). 2012;31(6):1277-85. DOI: 10.1377/ hlthaff.2011.0922

29. Lawson BJ, Burge FI, Mcintyre P, Field S, Maxwell D. Palliative care patients in the emergency department. J Palliat Care. United States; 2008;24(4):247-55.

30. Ouchi K, Wu M, Medairos R, Grudzen CR, Balsells $\mathrm{H}$, Marcus D, et al. Initiating Palliative Care Consults for Advanced Dementia Patients in the Emergency Department. J Palliat Med. 2014;17(3):346-50. DOI: 10.1089/jpm.2013.0285 